\title{
Development and Application of Identification Markers for Main Parental Lines of Hybrid Rice
}

Haiya Cai, Shuo Zhang, Gang Liu, Haitao Jia, Aiqing You ${ }^{\varpi}$, Chunhai Jiao $\leftleftarrows$

Institute of Food Crops, Hubei Academy of Agricultural Sciences, Hubei Key Laboratory of Food Crop Germplasm and Genetic Improvement, Wuhan, 430064, P.R. China

- Corresponding author email: aq you@163.com; jiaoch@hotmail.com

Rice Genomics and Genetics, 2022, Vol.13, No.1 doi: 10.5376/rgg.2022.13.0001

Received: 06 Jan., 2022

Accepted: 12 Jan., 2022

Published: 28 Jan., 2022

Copyright $\odot 2022$ Cai et al., This article was first published in Molecular Plant Breeding in Chinese, and here was authorized to translate and publish the paper in English under the terms of Creative Commons Attribution License, which permits unrestricted use, distribution, and reproduction in any medium, provided the original work is properly cited.

Preferred citation for this article:

Cai H.Y., Zhang S., Liu G., Jia H.T., You A.Q., and Jiao C.H., 2022, Development and application of identification markers for main parental lines of hybrid rice, Rice Genomics and Genetics, 13(1): 1-8 (doi: 10.5376/rgg.2022.13.0001)

\begin{abstract}
As the development of the modern hybrid rice breeding, many disadvantages behaved in variety identification, variety authenticity identification and seed genetic purity test based on phenotype, which was not suitable for the modern breeding. Insertion-Deletion (ID) loci in rice genome sequence were used to develop 9 ID markers to identify 29 hybrid rice backbone parental lines which were widely used in rice production. Cluster analysis of 29 parental lines were conducted using electrophoresis of those ID markers, and they were clustered into five groups with similarity coefficients of about 0.55 . In addition, according to relatedness and original region these lines were clustered into nine subgroups. Genetic purity was conducted for a hybrid rice Guangliangyou476 using ID marker and growth-out test methods, and similar results were generated. So molecular marker could be used into each stage of the modern breeding to accelerate breeding new variety. This research will be helpful for rice breeding, new cultivar registration and seed production.
\end{abstract}

Keywords Hybrid rice; Backbone parental line; Identification marker; Cluster analysis; Seed purity

Rice is the main staple food for a large segment of the world population (Zhang, 2007; Lin et al., 2019). Therefore, breeders have been pursuing genetic improvement of yield, quality, resistance and other traits of rice varieties. The number of new varieties breeding is also gradually increasing every year. For example, the number of rice varieties approved in China was 565 in 2008, and there were 943 by 2018, mainly were hybrid rice and indica rice, both of which exceeded 70\% (Lv, 2019, China Seed Industry, 2: 29-40). With the rapid increase in the number of approved varieties, higher requirements have been put forward for variety identification and diversity analysis.

DUS (Specificity, consistency and stability) determination should be carried out to identify the characteristics of the approved varieties. The traditional DUS determination of new rice varieties mainly rely on the identification of phenotypic parameters in the field. This method needs to record and observe the whole growth period of rice, which is time-consuming and has certain empiricism. Interaction between genotype and environment can also lead to phenotypic instability. In addition, in recent years, the repeated use of a few excellent backbone parental line has led to high genetic similarity of breeding varieties. It is difficult to distinguish these varieties by phenotype alone, and phenotype-based variety identification is far from meeting the requirements.

At present, the increase in the number of varieties in the market will also lead to the protection of intellectual property rights of varieties. The repeated use of a few excellent parents in modern breeding process leads to narrower genetic background and smaller phenotypic differences among varieties. For some varieties with large market demand, some similar counterfeit varieties will enter the market, resulting in intellectual property disputes in the sales process. Due to the high genetic similarity, it is difficult to distinguish such varieties by 
phenotype. Therefore, a new technology is urgently needed to solve the current problems in the protection of variety rights.

It is very important to monitor the purity of hybrid rice varieties in the production and sales process, because seed mixing will lead to the loss of rice quality and yield, resulting in disputes between farmers and sales enterprises. At present, two-line hybrid rice accounts for about half of the planting area of hybrid rice. Because the fertility transformation of two-line sterile lines depends on the natural environment (temperature and light), the decrease of temperature at booting stage in the process of seed production of two-line varieties will lead to the instability of fertility. In addition, genetic drift in the breeding process of the sterile line itself can also lead to fertility instability, resulting in self-fertilization of the sterile line and hybrid and purity reduction. The traditional purity detection used growth-out test method to determine the purity, and the purity was identified by phenotypic investigation after field planting. This method is time-consuming and labor-intensive, increasing operating costs for seed enterprises.

Molecular marker is a biotechnology developed in recent years and is widely used in crop genetics and other fields (Chen et al., 2009). Among them, the marker (Insertion Deletion, ID) designed according to the insertion deletion sequence of rice genome has the advantages of wide distribution, codominance, good repeatability, high polymorphism, stable amplification results and simple detection (Cai et al., 2017). In addition, compared with the latest array chip technology, molecular markers have the advantages of low technical cost and can be carried out in general small laboratories. Therefore, using ID markers to analyze the genetic diversity of the current backbone parental line of hybrid rice and distinguish the rice varieties currently used in production from the genomic level will become a new way to solve a series of problems in rice production mentioned above.

In this study, ID markers were developed to construct a set of marker groups that could separate the backbone parental line of some hybrid rice widely used in production at present. This group of markers identified 29 parents by the size difference of amplified bands. These markers were used for clustering analysis to construct the clustering map of these materials, and their application in purity detection of hybrid rice was explored, so as to provide help for hybrid rice production.

\section{Results and Analysis}

\subsection{Development of identification markers for backbone parental lines of hybrid rice}

A total of 29 selected backbone parental lines of hybrid rice widely used in rice production, including two-line sterile line, three-line sterile line and restorer line, were used to develop their detection marker combinations. Firstly, the genome sequences of sequenced varieties Nipponbare and 9311 were compared and primers were designed on both sides of the insertion and deletion sites. The principle of site selection was uniform distribution throughout the genome. 29 materials were amplified by PCR with ID primers, and the polymorphism index contents (PIC) of each pair of primers was calculated according to the PCR electrophoresis map. By selecting higher PIC primers, the tested varieties could be distinguished using the least marker combination. After sorting according to the size of PIC, the corresponding marker amplification band database was constructed (Figure 1), and finally 9 ID markers were selected to distinguish any variety from all other varieties (Table 1). At the same time, 9 marker electrophoresis maps constituted the identity number of the tested material varieties, that was fingerprint (Figure 1; Table 2). When distinguishing any variety, it is only necessary to select a combination of markers with different electrophoretic patterns between varieties. 
Rice Genomics and Genetics 2022, Vol.13, No.1, 1-8

http://cropscipublisher.com/index.php/rgg

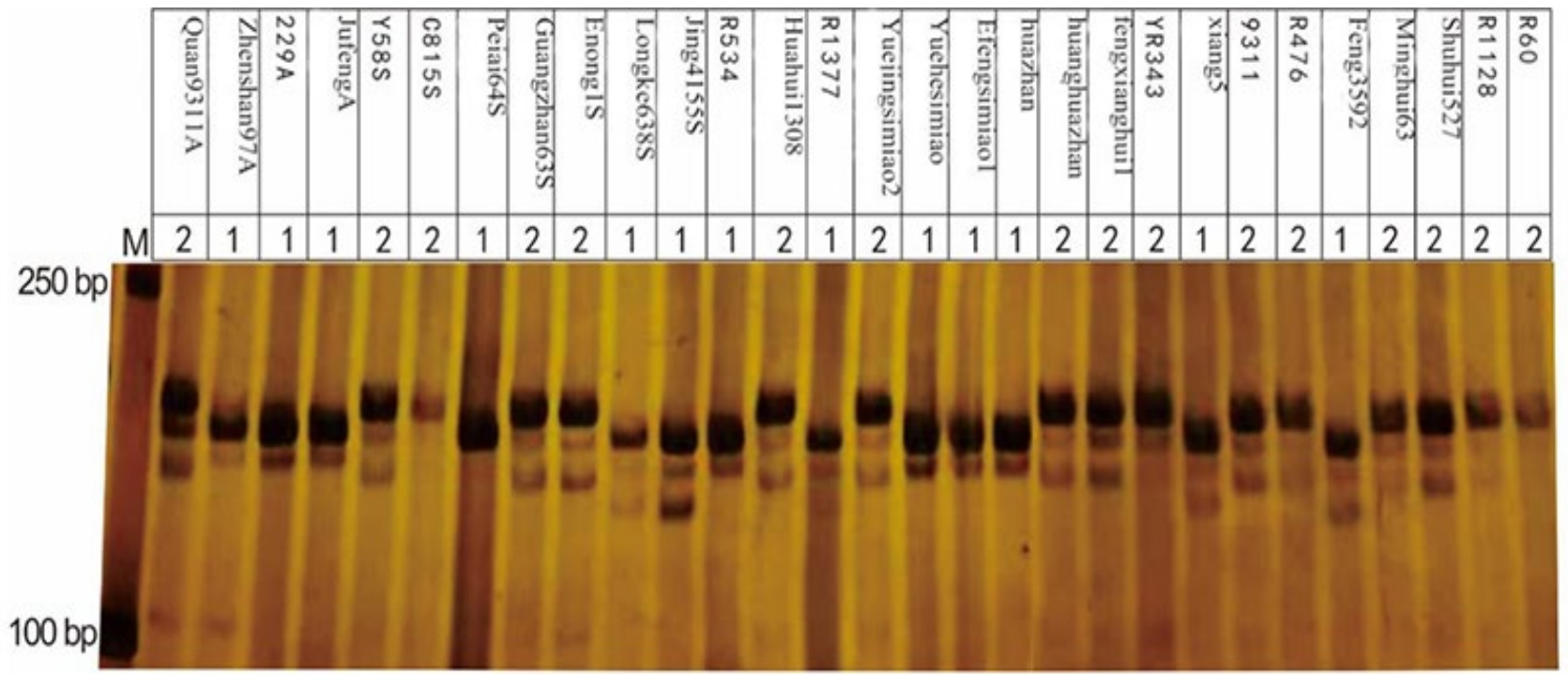

Figure 1 Electrophoresis of 29 hybrid rice parental lines for ID1 and their identity number construction

Note: M: DL2000 DNA Marker; Above the lane is corresponding variety of each lane, and the number is the identity number for each variety according to their electrophoresis

Table 1 Details of identification markers for 29 hybrid rice parental lines

\begin{tabular}{|c|c|c|c|c|c|}
\hline No. & ID marker & Marker sequence $\left(5^{\prime} \rightarrow 3^{\prime}\right)$ & Product length (bp) & $\operatorname{Tm}\left({ }^{\circ} \mathrm{C}\right)$ & PIC \\
\hline 1 & ID1 & $\begin{array}{l}\text { F: TGAGATGTGGCCATTAAGGA } \\
\text { R: GGCAAAAGATCTTATATTTACTTCG }\end{array}$ & 163 & 59 & 0.60 \\
\hline 2 & ID2 & $\begin{array}{l}\text { F: ACGGCTAAACGGTACTGCAT } \\
\text { R: ACACCAAGGGTGAAAAGTGG }\end{array}$ & 226 & 60 & 0.56 \\
\hline 3 & ID3 & $\begin{array}{l}\text { F: ACCTCATCATGCTGAACGTG } \\
\text { R: TGAGGAACTCCGACTTCTGG }\end{array}$ & 219 & 60 & 0.54 \\
\hline 4 & ID4 & $\begin{array}{l}\text { F: CAACTAAAACCAACACAAAATCCA } \\
\text { R: TGTCTAGTTGCATGTCTGAGTGTC }\end{array}$ & 130 & 60 & 0.52 \\
\hline 5 & ID5 & $\begin{array}{l}\text { F: CTCTGAGGTAGCAGCCATCG } \\
\text { R: TTAACCACACGCGGTTGC }\end{array}$ & 100 & 60 & 0.51 \\
\hline 6 & ID6 & $\begin{array}{l}\text { F: GAGTTCGGCGACAGTCAGT } \\
\text { R: TTGAAACATCCACGAATCTCA }\end{array}$ & 176 & 59 & 0.51 \\
\hline 7 & ID7 & $\begin{array}{l}\text { F: GGCAAGATTGGATTGAGGAG } \\
\text { R: TCGCCAAACGAAAAGAAAAT }\end{array}$ & 151 & 60 & 0.49 \\
\hline 8 & ID8 & $\begin{array}{l}\text { F: ATGGAACGCATGACATGAAA } \\
\text { R: CATCAAGAGGAGGGCAAAAA }\end{array}$ & 159 & 60 & 0.47 \\
\hline 9 & ID9 & $\begin{array}{l}\text { F: AATTCTTATGGACGGATACGC } \\
\text { R: TCAGCATCTCGTAAGCAAAAA }\end{array}$ & 159 & 58 & 0.47 \\
\hline
\end{tabular}

Note: F: Forward primer; R: Reverse primer; Tm: Annealing temperature; PIC: Polymorphism index contents; Product length is PCR product length in Nipponbare

\subsection{Cluster analysis}

Cluster analysis of 29 parental lines were conducted using UPGMA method, and the results showed (Figure 2) that they were clustered into 5 groups with similarity coefficients of about 0.55 . In addition, according to relatedness and original region these lines were clustered into 9 subgroups. For example, both subgroup I and subgroup II contain 9311 blood relatives, Enong1S in subgroup IV is bred by Guangzhan63S, and one of the parents of subgroup V 229A is JufengA. Besides, most varieties from the same region also clustered in the same subgroup. For example, most materials from Guangdong in subgroup III and subgroup VI clustered in the same subgroup 
Rice Genomics and Genetics 2022, Vol.13, No.1, 1-8

Table 2 Fingerprint construction of 29 test materials and their identification marker

\begin{tabular}{|c|c|c|c|c|c|c|c|c|c|c|c|}
\hline No. & Parental material & ID1 & ID2 & ID3 & ID4 & ID5 & ID6 & ID7 & ID8 & ID9 & Identification marker \\
\hline 1 & Quan9311A & 2 & 1 & 1 & 2 & 1 & 1 & 1 & 1 & 1 & ID1 ID8 \\
\hline 2 & Zhenshan97A & 1 & 1 & 1 & 1 & 2 & 1 & 1 & 1 & 2 & ID1 ID6 \\
\hline 3 & $229 \mathrm{~A}$ & 1 & 2 & 1 & 1 & 1 & 2 & 1 & 1 & 1 & ID1 ID9 \\
\hline 4 & JufengA & 1 & 2 & 1 & 1 & 1 & 2 & 1 & 1 & 2 & ID1 ID9 \\
\hline 5 & Y58S & 2 & 2 & 2 & 1 & 2 & 1 & 2 & 1 & 1 & $\mathrm{ID} 1 \sim \mathrm{ID} 8$ \\
\hline 6 & C815S & 2 & 2 & 2 & 1 & 2 & 1 & 2 & 2 & 2 & ID1 ID8 \\
\hline 7 & Peiai64S & 1 & 2 & 2 & 1 & 2 & 2 & 2 & 2 & 2 & ID1 ID5 \\
\hline 8 & Guangzhan63S & 2 & 2 & 2 & 1 & 2 & 2 & 1 & 1 & 2 & ID1 ID6 \\
\hline 9 & Enong1S & 2 & 1 & 1 & 1 & 2 & 2 & 1 & 1 & 2 & ID1 ID5 \\
\hline 10 & Longke638S & 1 & 2 & 1 & 1 & 1 & 1 & 2 & 1 & 2 & ID1 ID6 \\
\hline 11 & Jing4155S & 1 & 1 & 1 & 1 & 1 & 2 & 1 & 2 & 2 & ID1 ID5 \\
\hline 12 & R534 & 1 & 1 & 2 & 1 & 1 & 2 & 1 & 2 & 2 & ID1 ID4 \\
\hline 13 & Huahui1308 & 2 & 2 & 2 & 2 & 2 & 1 & 2 & 2 & 2 & ID1 ID5 \\
\hline 14 & $\mathrm{R} 1377$ & 1 & 2 & 2 & 1 & 1 & 2 & 2 & 1 & 2 & ID1 ID5 \\
\hline 15 & Yuejingsimiao2 & 2 & 2 & 2 & 2 & 1 & 1 & 2 & 1 & 2 & ID1 ID5 \\
\hline 16 & Yuehesimiao & 1 & 1 & 2 & 2 & 2 & 1 & 2 & 1 & 2 & ID1 ID4 \\
\hline 17 & Efengsimiao1 & 1 & 1 & 1 & 2 & 2 & 1 & 1 & 1 & 2 & ID1 ID6 \\
\hline 18 & Huazhan & 1 & 1 & 1 & 2 & 2 & 2 & 1 & 1 & 2 & ID1 ID6 \\
\hline 19 & Huanghuazhan & 2 & 1 & 1 & 2 & 1 & 1 & 1 & 2 & 2 & ID1 ID8 \\
\hline 20 & Fengxianghui1 & 2 & 2 & 2 & 1 & 1 & 1 & 1 & 1 & 1 & ID1 ID6 \\
\hline 21 & YR343 & 2 & 1 & 2 & 2 & 1 & 1 & 1 & 1 & 1 & ID1 - ID4 \\
\hline 22 & Xiang5 & 1 & 2 & 2 & 2 & 1 & 1 & 1 & 1 & 1 & ID1 ID4 \\
\hline 23 & 9311 & 2 & 2 & 1 & 2 & 1 & 1 & 1 & 1 & 1 & ID1 ID8 \\
\hline 24 & R476 & 2 & 1 & 1 & 2 & 1 & 2 & 1 & 1 & 1 & ID1 ID6 \\
\hline 25 & Feng3592 & 1 & 1 & 1 & 1 & 2 & 2 & 2 & 2 & 1 & ID1 ID6 \\
\hline 26 & Minghui63 & 2 & 1 & 1 & 1 & 1 & 2 & 2 & 2 & 1 & ID1 ID5 \\
\hline 27 & Shuhui527 & 2 & 2 & 1 & 1 & 2 & 1 & 2 & 1 & 1 & ID1 ID4 \\
\hline 28 & $\mathrm{R} 1128$ & 2 & 2 & 2 & 1 & 1 & 2 & 2 & 2 & 1 & ID1 ID6 \\
\hline 29 & R60 & 2 & 1 & 2 & 1 & 2 & 2 & 2 & 2 & 2 & ID1 ID4 \\
\hline
\end{tabular}

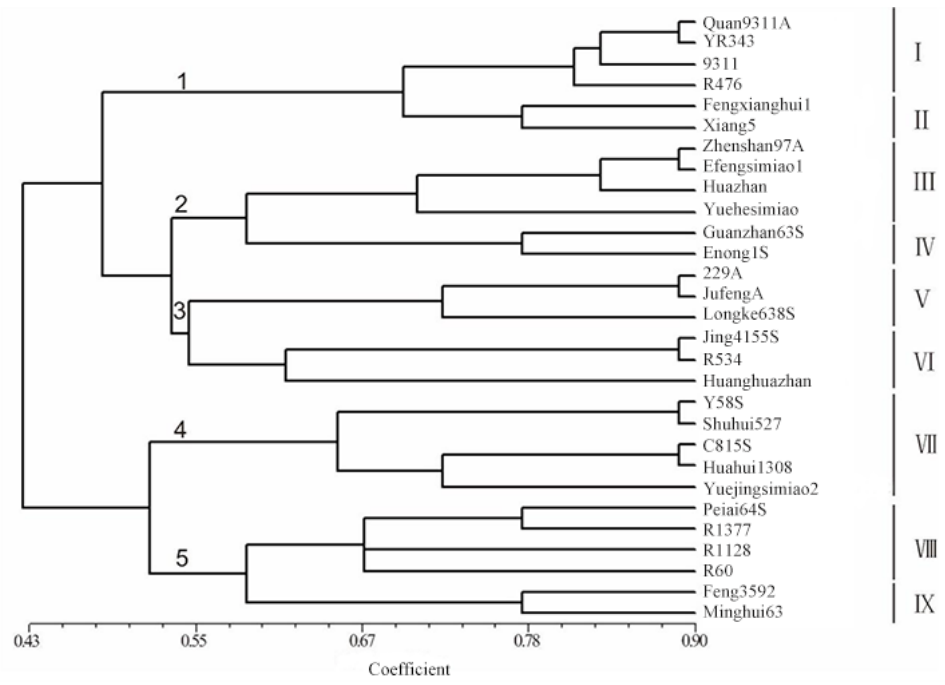

Figure 2 Dendrogram of genetic relationships among 29 rice varieties by UPGMA cluster analysis Note: Scale at the bottom is coefficient of similarity 


\subsection{Detection of hybrid rice seed purity by identification markers}

Two-line hybrid rice Guanglaingyou476 with the parents of Guangzhan63S and R476 was selected to detect purity. According to the parental identity number of hybrid rice in Table 2, the corresponding value of Guangzhan63S identity number in ID3 is 2 . The corresponding value of R476 ID number in ID3 is 1 . Therefore, ID3 polymorphism between the two parents can distinguish the two, so ID3 was selected to detect the purity of the hybrid. A total of 74 individual plant samples were detected, of which three bands were homozygous female parental bands, and the rest were heterozygous bands from parents (Figure 3). Based on this, it was speculated that the purity of the seeds in this batch was about $95.9 \%$. At the same time, 500 seeds of this batch were planted in the field. The identification results of grow-out test showed that all the heterozygous plants were female parent with the purity of $96.1 \%$, which was basically consistent with the results of marker detection. Therefore, the purity of hybrid rice seeds can be detected by molecular markers.

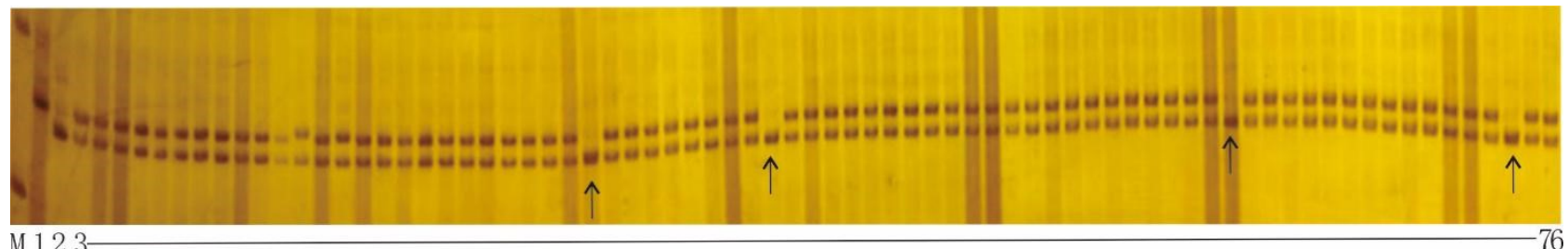

M 123

Figure 3 Genetic purity test of two-line hybrid rice Guanglaingyou476 using ID3 marker

Note: M: DL2000 DNA Marker, lane 1: Male parent R476; lane 2: Female parent Guangzhan63S; and all other 74 are random selected seeds

\section{Discussion}

In this study, the development of identification markers for 29 common backbone parental lines of hybrid rice was reported, and the fingerprints of these materials were constructed according to the marker electrophoresis. And cluster analysis of test materials was conducted. Finally, the seed purity of a hybrid rice was identified with the identification markers.

With the acceleration of modern breeding process, crop varieties have increased dramatically. The genetic background between different varieties is becoming narrower and narrower, and the phenotypic differences are becoming smaller and smaller. Therefore, the identification and distinction of varieties based on phenotype have become more difficult. Molecular markers have the characteristics of wide variation, genetic stability and partial co-dominance. Therefore, DNA fingerprinting has great advantages in identifying the characteristics of varieties at the genomic level (Akkaya et al., 1992; Nandakumar et al., 2004). At present, the International Union for the Protection of New Varieties (UPOV) and the Ministry of Agriculture of the PRC have added molecular markers to DUS determination. In this study, 9 ID markers were developed, and 29 varieties could be distinguished by one or more combinations of these markers. At the same time, the offspring selected by these backbone parental lines also could be distinguished. Therefore, this method could effectively compensate for the lack of phenotypic identification materials. In recent years, anti-counterfeiting incidents occurred frequently in the seed market. For some varieties with large market demand, there will be some counterfeit varieties with similar phenotypes entering the market, resulting in intellectual property disputes during the sales process. Therefore, these varieties can be distinguished by the variety-specific markers identified in this study, thus protecting the equity of breeders.

In recent years, there have been frequent anti-counterfeiting incidents in the seed market. For some varieties with large market demand, there will be some counterfeit varieties with similar phenotypes entering the market, resulting in intellectual property disputes during the sales process. Therefore, these varieties can be distinguished by the variety-specific markers identified in this study, thus protecting the rights and interests of breeders. 
Xiao et al. (1996) found that the yield potential and its heterosis were positively correlated with the genetic distance between parents in indica and japonica rice. This view has also been confirmed in other crops (Corbellini et al., 2002). Therefore, genetic differences among varieties can be intuitively seen by constructing phylogenetic tree among varieties through cluster analysis, which can provide useful help for new variety breeding and hybrid combination selection (Joshi et al., 2001; Guo et al., 2012; Hu et al., 2016). In this study, the developed markers were used to construct the fingerprint of the tested materials, which can provide useful reference for breeding workers.

The production and sales of hybrid rice varieties must meet certain purity requirements, and the decrease of purity will lead to the decline of rice quality and yield. Weather changes during seed production of two-line sterile lines can lead to fertility restoration and self-fruiting. Besides, the genetic drift in the breeding process of the sterile line itself can also lead to fertility instability, and ultimately lead to the decrease of hybrid purity. In this study, the purity identification of a two-line hybrid rice variety by molecular markers and field tests showed that the detected hybrids were female parent, and the purity was basically the same. Therefore, molecular markers can replace field sequencing for purity identification.

\section{Materials and Methods}

\subsection{Test materials}

In this study, 29 backbone parental lines of indica rice widely used in rice production in China were selected, including 4 three-line sterile lines, 7 two-line sterile lines and 18 restorer lines. Hybrid rice Guanglaingyou 476 with the parents of Guangzhan63S and R476 was selected to detect purity. The seeds were commercial varieties purchased from the market. And the parent materials were provided by the Institute of Food Crops, Hubei Academy of Agricultural Sciences, and strictly eliminated in the field. The field growth test of two-line hybrid rice was used to identify the hybrid degree of sterile lines by observing whether the individual plant was seed.

\subsection{DNA extraction, PCR amplification and PCR product detection}

When extracting DNA from parent materials, 3 leaves of single plant were selected and mixed, and DNA of single plant was extracted when hybrid rice seeds grew to 3-leaf stage. DNA was extracted by CTAB method (Murry and Thompson, 1980), and the DNA concentration was finally adjusted to $30 \mathrm{ng} / \mu \mathrm{L}$ for further use. The PCR amplification system was $20 \mu \mathrm{L}$, including template DNA $2.0 \mu \mathrm{L}, 25 \mathrm{mmol} / \mathrm{L}$ buffer $2.0 \mu \mathrm{L}, 10 \mathrm{mmol} / \mathrm{L}$ primer $1.0 \mu \mathrm{L}, 2.5 \mathrm{mmol} / \mathrm{L}$ dNTP $1.0 \mu \mathrm{L}, 5 \mathrm{U} / \mu \mathrm{L}$ Taq DNA polymerase $0.2 \mu \mathrm{L}$, $\mathrm{ddH}_{2} \mathrm{O} 13.8 \mu \mathrm{L}$. PCR reaction program was as follows: pre-denaturation for $5 \mathrm{~min}$ and denaturation for $45 \mathrm{~s}$ at $94^{\circ} \mathrm{C}$, annealing temperature according to different primers, annealing time for $45 \mathrm{~s}, 72^{\circ} \mathrm{C}$ extension for $45 \mathrm{~s}, 30 \mathrm{cycles}$, and the final product was extended at $72^{\circ} \mathrm{C}$ for $8 \mathrm{~min}$ and stored in $4{ }^{\circ} \mathrm{C}$ refrigerator. PCR products were added with $4 \mu \mathrm{L}$ sample buffer, and $2 \mu \mathrm{L}$ products were electrophoresed on $6 \%$ polyacrylamide gel (200 V, 90 min). After electrophoresis, the gel was stripped from the glass, placed in silver staining solution $\left(0.2 \mathrm{~g} / \mathrm{L} \mathrm{AgNO}_{3}\right)$ and shaken for $8 \mathrm{~min}$, and then rinsed with distilled water for $20 \mathrm{~s}$. After that, the gel was placed in the developer $(15.0 \mathrm{~g} / \mathrm{L} \mathrm{NaOH}, 0.5 \%$ formaldehyde) until the bands were clearly visualized. Finally, took photos in the light box and performed the statistical data.

\subsection{Primer design}

The genome sequences of sequenced japonica rice Nipponbare and indica rice 9311 were used for comparison, and the sites with InsertDelet (ID) sequences between $6 \sim 15 \mathrm{bp}$ were selected, and the primer premier 5.0 was used to design the ID primers on both sides. The site selection principle was uniform distribution in the whole genome. 29 materials were amplified by PCR using ID primers, and the polymorphism index contents (PIC) of each pair of primers was calculated according to the PCR electrophoresis. The calculation formula was as follows: $\mathrm{PIC}=1-\Sigma(\mathrm{Pi})^{2}$, where $\mathrm{Pi}$ was the gene frequency of the insertion loss primer at the $\mathrm{i}^{\text {th }}$ polymorphic site. By selecting higher PIC primers, the least marker combinations can be used to distinguish the tested varieties. 


\subsection{Fingerprint construction}

The obtained ID markers were arranged according to the PIC value from large to small. According to the molecular weight of the bands in the electrophoresis of each marker, the Arabic digital number of each complex allele site was carried out from large to small, and the corresponding band type values of each variety were obtained. The combination of band type values of multiple ID markers in each variety constitutes the SSR fingerprint of the variety. When 9 ID markers were selected, all varieties obtained the unique fingerprint. Therefore, any parent can be separated from other parents by using these 9 ID markers.

\subsection{Cluster analysis}

According to the electrophoresis, the database of ID marker amplification band type was constructed. The ID marker amplification products were assigned to " 0 " and " 1 ". At the same mobility position, if there was electrophoresis band record "1", if there was no record "0" the Excel data was constructed. NTsys 2.1 software was used to calculate the genetic similarity coefficient. According to the genetic similarity coefficient, the cluster analysis was carried out by un-weighted pair-group method of arithmetic means (UPGMA) and the clustering map was drawn.

\section{Authors' contributions}

CHY, ZS are the experimental designers and executors of this research. LG completed the data analysis and manuscript writing. JHT participated in the design of the study and performed the statistical analysis. YAQ and JCH are the designer and director of the project, guiding the experimental design, data analysis, writing and revision. All authors read and approved the final manuscript.

\section{Acknowledgments}

This study was supported by the Innovation Project of Hubei Agricultural Science and Technology Innovation Center "Protection and Utilization of crop Germplasm Resources and Germplasm Innovation” (2019-620-000-001-01).

\section{Reference}

Akkaya M.S., Bhagwat A.A., and Cregan P.B., 1992, Length polymorphisms of simple sequence repeat DNA in soybean, Genetics, 132(4): 1131-1139 https://doi.org/10.1093/genetics/132.4.1131 PMid:1459432 PMCid:PMC1205234

Cai H.Y., Zhou L., Jiao C.H., Fu Y.Z., You A.Q., and Xu D.Z., 2017, Development and application of rice blast resistant gene Pita gene-specific molecular marker, Fenzi Zhiwu Yuzhong (Molecular Plant Breeding), 15(2): 589-593

Chen Z.G., Deng H.C., and Zhao S.P., 2009, Genetic diversity and relationship of 47 rice varieties revealed by ISSR, Jiyinzuxue Yu Yingyong Shengwuxue (Genomics and Applied Biology), 28(3): 498-502

Corbellini M., Perenzin M., Accerbi M., Vaccino P., and Borghi B., 2002, Genetic diversity in bread wheat, as revealed by coefficient of parentage and molecular markers, and its relationship to hybrid performance, Euphytica, 123(2): 273-285 https://doi.org/10.1023/A:1014946018765

Guo J.H., Li X.G., Li L., Li S.P., Luo X., Wei S.X., and Xie Z.S., 2012, Based on the ISSR analysis of genomic DNA diversity of 28 banana germplasms, Jiyinzuxue Yu Yingyong Shengwuxue (Genomics and Applied Biology), 31(05):492-497

Hu Y., Li Y., Huang Y.Z., and Liu P.W., 2016, Genetic diversity evaluation of sugarcane varieties using SSR and RAPD markers, Jiyinzuxue Yu Yingyong Shengwuxue (Genomics and Applied Biology), 35(09): 2494-2503

Joshi S.P., Bhave S.G., Chowdari K.V., Apte G.S., Dhonukshe B.L., Lalitha K., and Gupta V.S., 2001, Use of DNA markers in prediction of hybrid performance and heterosis for a three-line hybrid system in rice, Biochem. Genet., 39(5-6): 179-200 https://doi.org/10.1023/A:1010293325482 PMid:11530855

Lin Z.S., Liu G.M., and Xu Q.G., 2019, Analysis of genetic diversity of new rice lines by SRAP markers, Jiyinzuxue Yu Yingyong Shengwuxue (Genomics and Applied Biology), 38(4): 1683-1688

Murry M.G., and Thompson W.F., 1980, Rapid isolation of high molecular weight plant DNA, Nucleic Acids Res., 8(19): 4321-4325 https://doi.org/10.1093/nar/8.19.4321 PMid:7433111 PMCid:PMC324241

Nandakumar N., Singh A.K., Sharma R.K., Mohapatra T., Prabhu K.V., and Zaman F.U., 2004, Molecular fingerprinting of hybrids and assessment of genetic purity of hybrid seeds in rice using microsatellite markers, Euphytica, 136: 257-264 https://doi.org/10.1023/B:EUPH.0000032706.92360.c6 
Rice Genomics and Genetics 2022, Vol.13, No.1, 1-8

$\mathrm{http} / / /$ cropscipublisher.com/index.php/rgg

Xiao J., Li J., Yuan L., McCouch S. R., and Tanksley S.D., 1996, Genetic diversity and its relationships to hybrid performance and heterosis in rice as revealed by PCR-based markers, Theor. Appl. Genet., 92: 637-643

https://doi.org/10.1007/BF00226083

PMid:24166385

Zhang Q.F., 2007, Strategies for developing green super rice, Proc. Natl. Acad. Sci., 104(42): 16402-16409

https://doi.org/10.1073/pnas.0708013104

PMid:17923667 PMCid:PMC2034246 